\title{
DIVIMP simulation of W transport in the SOL of JET H-mode plasmas
}

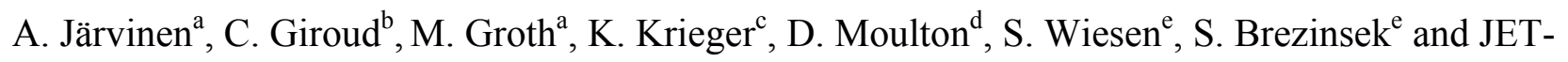
EFDA contributors ${ }^{1}$

JET-EFDA, Culham Science Centre, Abingdon, OX14 3DB, UK

${ }^{a}$ Aalto University, Association EURATOM-Tekes, P.O.Box 4100, 02015 Espoo, Finland

${ }^{b}$ EURATOM/CCFE Fusion Association, Culham Science Centre, Abingdon, Oxon. OX14 3DB, UK.

${ }^{C}$ max-Planck-Institut für Plasmaphysik, EURATOM-Assoziation, D-85748 Garching, Germany

${ }^{d}$ Imperial College of Science, Technology and Medicine, London, UK

'IEF-Plasmaphysik, Forschungszentrum Jülich, Association EURATOM-FZJ, Jülich, Germany

${ }^{1}$ See the Appendix of F. Romanelli et al., Proceedings of the 23rd IAEA Fusion Energy Conference 2010, Daejeon, Korea

PACS: 52.25.Vy; 52.40.Hf; 52.65.Pp

\section{Abstract}

This study provides predictions of tungsten contamination extrapolated from an inter-ELM phase of a high-triangularity, high-confinement, ITER-like wall reference plasma without impurity seeding in JET. The tungsten concentration for an equivalent ILW plasmas are predicted for low, medium, and high upstream densities, utilising the Monte Carlo trace-impurity code DIVIMP on background plasmas calculated with the 2-D fluid code EDGE2D/EIRENE. It is observed that operation at the low upstream density plasma with a sheath-limited scrape-off layer leads to core tungsten concentration of the order $10^{-5}$. Increasing the density leads to high-recycling scrape-off layer with adequate divertor retention to ensure acceptable tungsten core concentration below $10^{-5}$. Sufficient divertor retention is achievable when the peak target electron temperature, $T_{e}$, is below $10 \mathrm{eV}$. Achieving significant reduction of tungsten sputtering by multiple charged impurity species (e.g., $\mathrm{Be}^{2+}, \mathrm{C}^{4+}$ ) requires a peak target $T_{e}$ below $5 \mathrm{eV}$, which requires strong divertor radiation achieved by impurity seeding in the ILW configuration.

\section{Introduction}

The performance of future fusion reactors highly depends on the impurity content of the confined plasma. Plasma impurities decrease the performance both by diluting the plasma and increasing the core radiation losses, thus decreasing the fusion reaction rate and cooling the plasma core. High-Z impurities, such as tungsten (W), have a particularly significant effect, as they lead to intense 
radiation in the core relevant temperatures in the range of $\mathrm{keV}$. Hence, tungsten concentration, $c_{w}$, below several $10^{-5}$ is required for the core of reactor relevant plasmas [1]. Despite its detrimental effect to the core plasma, tungsten is favored as the divertor target material, instead of carbon, during the ITER deuterium-tritium (DT) phase due to low fuel retention and low physical sputtering at semi-detached conditions [2]. To address the effect of the PFC material on the fuel retention and the consequence of absence of the main intrinsic radiator in the divertor, the carbon-fiber composite tiles in the JET tokamak are currently being replaced with beryllium in the main chamber and tungsten in the divertor $[3,4]$.

This study gives a predictive estimate of tungsten target sputtering and transport during an interELM phase of an unseeded and almost unfuelled JET ITER-like wall reference plasma of hightriangularity: JPN \#76666, H-mode confinement, $B_{t}=2.7$ T, $I_{p}=2.5 \mathrm{MA}, P_{i n} \sim 16 \mathrm{MW}, \delta \sim 0.4$ [5]. This plasma is ILW-compatible with respect to steady state power loads, but incompatible with respect to electron temperature in the divertor and during ELMs if no mitigation techniques are applied. The background plasmas were simulated with the fluid code EDGE2D/EIRENE [6,7], producing 2-D maps of $n_{e}, T_{e}, T_{D+}$, flow $V$, and $E_{\|}$, and validated against core and edge measurements in JET [8]. Based on these background plasmas, W erosion and transport is simulated with the Monte Carlo code DIVIMP coupled with EIRENE [9, 7].

\section{Simulation details}

The anticipated operating regimes for plasma in the ITER-like wall configuration were assessed with EDGE2/EIRENE by performing a fuelling scan matching the three experimental conditions obtained in JPN 76666 and the associated pulse series [5, 8]. The background plasma solutions included carbon sputtered from the main chamber wall and divertor plates, and the density was controlled by adjusting inner target deuterium puff as in the experiments: low density (fuelling = $5 * 10^{21}$ electrons/s), medium density $\left(11 * 10^{21}\right.$ electrons/s), and high density $\left(14 * 10^{21}\right.$ electrons/s) 
[8]. The puffing location is shown in [8]. For the low-density case, fuelling was adjusted to match the outer mid plane $n_{e}$ and $T_{e}$ profiles, and boundary conditions at the divertor plates (e.g., chemical sputtering yields) were set to match the outer target $J_{s a t}, T_{e}$ measured by Langmuir probes, and infrared camera heat load $\left(Q_{I R}\right)$ [8]. The medium and high-density cases are obtained from the lowdensity case by scaling the inner target fuelling [8].

The primary tungsten sputtering in DIVIMP is calculated by multiplying the $\mathrm{D}^{+}$influx towards target with sputtering yield obtained from Eckstein 1993 yield formula [10] with an assumption of $1 \%$ helium-like carbon $\left(\mathrm{C}^{4+}\right)$ contamination in the plasma, which is an empirical assumption from ASDEX Upgrade [11]. Initial sputtering only due to $\mathrm{C}^{4+}$ is assumed. The impact energy is given by $E_{\text {imp }}=2 T_{b}+3 T_{e} Z_{\text {imp }}$

where $Z_{\text {imp }}=4$, and $T_{b}$ and $T_{e}$ are the local background plasma ion and electron temperatures. The assumption of $\mathrm{C}^{4+}$ contamination represents the presence of light impurities, such as residual carbon, impurities from plasma seeding, and main chamber beryllium. The tungsten contamination given by DIVIMP scales linearly in the magnitude of the source, and thus, an order of magnitude estimate of tungsten contamination for plasmas with impurity fraction of $1-3 \%$ in the divertor can be obtained by scaling those from the $1 \%$ contamination case. The enhancement of sputtering due to the non-normal incidence has not been taken into account. Self-sputtering and prompt re-deposition models of DIVIMP were used. In the case of prompt re-deposition, a particle will re-deposit to the wall during its first gyro orbit if it ionizes within the distance of its larmour radius from the wall. If the particle is ionized within the magnetic pre-sheath, the impact energy is reduced accordingly.

In DIVIMP tungsten transport is calculated in the test particle limit using parallel-B force equation and perpendicular-B anomalous diffusion [9]. The target sputtered neutrals are followed to their ionization location using neutral code EIRENE. The initial velocity, $v$, and angle to the target normal, $\theta$, of sputtered neutrals are given by:

$\theta=\tan ^{-1}(\tan (\beta) \cos (\varphi))$ 
$v=\sqrt{\frac{2 E_{\text {bind }}}{M_{i}} \frac{1}{\frac{1}{\sqrt{\gamma}}-1}} \sqrt{\left(\cos (\beta)^{2}+\left(\sin (\beta)^{2} \cos (\varphi)^{2}\right)\right)}$

where $\beta=\sin ^{-1}(\sqrt{\alpha}), \varphi=2 \pi \eta, E_{b i n d}$ is the binding energy of the target material, and $M_{i}$ the atomic mass of the target material. The parameters $\alpha, \eta \in[0,1]$ and $\gamma \in[0, \max ]$ are uniform random numbers, where max corresponds to the maximum energy of sputtered particles. The parallel-B force includes impurity pressure gradient, background friction, electrostatic force, and temperature gradient forces due to electrons and ions. The impurity pressure gradient force in DIVIMP is modelled as parallel-B diffusion. The parallel-B forces are modelled with fluid approximation in this study. An additional simulation with drift kinetic model for parallel forces, according to [12], is performed for comparison purposes. For perpendicular-B motion, a diffusion coefficient $\mathrm{D}_{\lrcorner}$of 1 $\mathrm{m}^{2} / \mathrm{s}$ is used in this study. Ionization and recombination are calculated using ADAS database 1997 for tungsten [13 ]. Impurity particles experience finite thermalization relative to the background $T_{D^{+}}$. In this study, the evolution of approximately 100000 test particles is calculated to provide a steady-state solution of tungsten density in the computational domain.

\section{W target sputtering and SOL transport}

Table 1 presents the background plasma $n_{e}, T_{e}, T_{i}$, at targets and mid planes, total deuterium current,

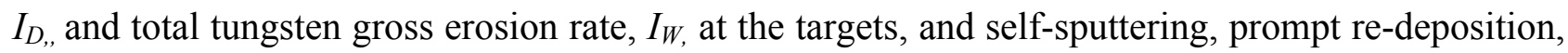
and leakage fractions, as well as the tungsten contaminations values in core, main SOL and divertor SOL for the three simulated background plasmas. The inner target is predicted to be high-recycling or detached giving low target temperature and, thus, low physical sputtering of tungsten. Inner target provides $16 \%$ of the tungsten sputtering in the lowest density plasma and $0 \%$ in the medium and high density plasmas (Table 1). The outer target scrape-off layer is predicted to be sheathlimited in the low-density case with peak target $T_{e} / T_{i}$ around 107/92 eV, and high-recycling in the medium and high-density cases with peak target $T_{e} / T_{i}$ values around 8/5.5 eV and 4/5.3 eV between 
ELMs [8]. The tungsten sputtering is highly dominated by physical sputtering at the outer strike point, where the peak target $T_{e}$ is observed (Fig. 1). The $I_{W}$ is reduced by two orders of magnitude when going from low to high density (Table 1). Reducing the peak target $T_{e}$ from $107 \mathrm{eV}$ to $8 \mathrm{eV}$ provides only a factor of 4 reduction of tungsten sputtering due to $\mathrm{C}^{4+}$, whereas reducing the peak $T_{e}$ further down to $4 \mathrm{eV}$ provides 2 orders of magnitude difference to the low density case because the targets are then almost entirely below the physical sputtering threshold for $\mathrm{C}^{4+}$ ions on tungsten. In the low density case with peak target $T_{e}$ around $107 \mathrm{eV}$, significant physical sputtering due to $\mathrm{D}^{+}$ would also occur. An additional simulation shows that ignoring tungsten sputtering due to $\mathrm{D}^{+}$in the low-density case gives a factor of 1.7 reduction of the source. The self-sputtering gives around $20 \%$ of sputtering for the low density case and almost negligible below $1 \%$ of sputtering for the medium and high density cases. The fraction of self-sputtering depends, however, on the fraction of prompt re-deposition. Without prompt re-deposition, the related self-sputtering fractions would be $60 \%$ and $6-3 \%$ of the total tungsten sputtering. The prompt re-deposition fraction is significant. The fraction is calculated by dividing the number of prompt re-deposited test particles with the total number of launched neutral tungsten test particles. In the low-density plasma around $80-90 \%$ of sputtered tungsten redeposit promptly back to the target plates. In the medium and high density plasmas, the fraction is $97-99 \%$.

The core (inside separatrix) concentration of tungsten is around a few $10^{-5}$ for the low density plasma and thus, at the threshold of acceptable contamination (Fig. 2, Table 1). As the density is increased, the core concentration decreases rapidly being around $10^{-11}$ for the medium density case and zero, within the computational accuracy, for the high density case. The main scrape-off layer contamination shows similar behaviour compared to the core contamination, but is approximately 1 to 2 orders of magnitude higher than the associated core concentration. A diffusion coefficient scaling with $\mathrm{D}_{\perp}=2 \mathrm{~m}^{2} / \mathrm{s}$ and $10 \mathrm{~m}^{2} / \mathrm{s}$ gives variation of the contamination within one order of magnitude. An additional simulation shows that utilizing drift kinetic model instead of fluid 
approximation for parallel forces leads to similar results, the core contamination values being $3.8 * 10^{-5}$ (low density), $1.4 * 10^{-10}$ (medium density), and 0 (high density).

The tungsten divertor retention is significantly increased with increasing upstream density. The percentage of gross-eroded particles reaching the core plasma is around $3.5 \%$ for the low density case, $0.0004 \%$ for the medium density case and $0 \%$ for the high density case. The parallel-B transport properties in divertor leg area are dominated by the balance between friction force and ion temperature gradient force, which in fluid approximation can be written [14]:

$$
\begin{gathered}
F_{f r i c}=m_{W} \frac{v_{D}-v_{W}}{\tau_{s}} \\
F_{\nabla T_{i}}=\beta \nabla T_{i}
\end{gathered}
$$

where $\tau_{\mathrm{s}}$ is the Spitzer stopping time, $\beta$ is a coefficient depending on masses of the impurity and background ions and on the charge state of the impurity ion $\left(\beta \approx 260\right.$, for $\left.\mathrm{W}^{10+}\right)$. In divertor leg region the friction force (eq. 4) tends to point towards target and the ion temperature gradient force (eq. 5) towards upstream. As $\tau_{\mathrm{s}} \propto T^{3 / 2} n^{-1}{ }_{D}$, the ratio of these forces can be written

$$
\left(\begin{array}{c}
F_{\text {fric }} \\
F_{\nabla T_{i}}
\end{array}\right) \propto \frac{\left(v_{D}-v_{Z}\right) n_{D}}{T_{D}^{3 / 2} \frac{d T_{D}}{d s}},
$$

where $T_{D}$ represents the background temperature, $s$ the coordinate parallel-B, and $n_{D}$ the background deuterium density. Thus, the interesting plasma background quantities in terms of divertor leakage are $v_{D^{+}}, n_{D^{+}}, T_{D}$, and $d T_{D} / d s$. If the ratio (eq. 6) is above 1 , friction force dominates giving high divertor retention.

This paragraph analyses the background plasma $v_{D^{+}}, n_{D^{+}}, T_{D}$, and $\tau_{s}$ upstream from outer strike point (OSP) to low field side (LFS) x-point, in order to investigate the effect of background plasma profile to tungsten divertor retention. The increased upstream density leads to highrecycling conditions at the outer strike zone cooling down the plasma at the divertor region by a 
factor of 3-4 compared to the low density case (Fig.3 a). The ion temperature gradient steepens near the target plate, but becomes shallower further upstream. Thus, the ion temperature gradient force is reduced with increased upstream density along most of the outer divertor leg. The divertor density increases approximately by a factor of 4 with increasing upstream density (Fig. 3b). The increased divertor density together with reduced ion temperature leads to a significant increase in divertor collisionality, as the Spitzer stopping time $\tau_{\mathrm{s}} \propto T^{3 / 2} n^{-1}$ (Fig. 3c). The Spitzer stopping time is around 1.5 orders of magnitude lower in higher density plasmas compared to the low density case along most of the divertor region upstream from OSP. The background plasma flow velocity is just at the plate by a factor $2-5$ higher in low density plasma compared to medium and high density plasmas (Fig. 3d). The medium density plasma gives lowest flow velocity. The high-recycling cases give flow reversal around $10 \mathrm{~m}$ away from the target along magnetic field line. The increased collisionality dominates over the reduced background flow velocity, thus increasing the friction force towards target with reducing divertor plasma temperature. The change in ion temperature gradient force is not as great as the change in background friction. Thus, tungsten divertor retention is greatly enhanced with increasing fuelling as a result of increased friction force due to increased collisionality.

\section{Discussion}

This study estimated concentration of target-sputtered tungsten in JET H-mode plasma assuming steady-state inter-ELM condition. Assuming steady-state inter-ELM plasma is the first order approximation in understanding impurity transport and core contamination in an H-mode plasma. In reality, the plasma is dynamically evolving with the ELM frequency $\left(f_{E L M}\right)$ of the order of $10 \mathrm{~Hz}$ revealing the simplicity of the used approximation. The model presented here does not take into account the high peak target sputtering during ELMs, which is anticipated to be the dominant source of tungsten in JET ITER-like wall operation [15]. On the other hand, the ELM caused flushing of impurities out of core is also ignored. 
In these studies, the peak target $T_{e}$ drops from around $100 \mathrm{eV}$ down to $10 \mathrm{eV}$ between low and medium density plasmas. From the modelling point of view, intermediate steps in density would have been necessary to elucidate transition regimes.

The predicted peak target temperature $107 \mathrm{eV}$ is above both single and triple Langmuir probe measurements. It is anticipated that the real peak target temperature would be within the range of 35-85eV given by the Langmuir probe measurements [8]. Thus, the prediction of tungsten contamination for the low-density case can clearly be taken as a worst case scenario for a steadystate inter-ELM plasma. During ELMs these temperature ranges are, however, expected.

\section{Summary}

The predicted core $\mathrm{W}$ concentration, $c_{w}$, is calculated for three different densities in a hightriangularity, high-confinement ITER-like wall reference case in JET. Tungsten transport calculations were performed with DIVIMP utilising EDGE2D/EIRENE background plasma simulations. According to these calculations, $c_{w}$ exceeds $10^{-5}$ on the entire computational domain in the low density case, in which the SOL is in the sheath-limited regime. Given the EDGE2D/EIRENE predicted $T_{e}$ of $100 \mathrm{eV}$, and higher, in front of the outer target plate, this case is evidently the worst case for inter-ELM periods. Increasing the upstream density to the medium case leads to a sufficient divertor retention of $\mathrm{W}$, as the plasma conditions at the outer strike point become high-recycling and the background plasma couples collisionaly better to $\mathrm{W}$ ions. For interELM plasma conditions $c_{w}$ falls below $10^{-7}$, while the target sputtering source decreases only by a factor of 4 compared to the low-density plasma. Increasing the density to the highest case leads to around 2 orders of magnitude reduction in the tungsten sputtering, which is associated with reduction of peak target $T_{e}$ below $5 \mathrm{eV}$. Thus, it can be concluded that in high-recycling inter-ELM conditions with peak target $T_{e}$ below $10 \mathrm{eV}$ the core $c_{w}$ below $10^{-7}$ is achievable without significant reduction of tungsten source. To reduce the tungsten sputtering significantly, peak target $T_{e}$ below 5 $\mathrm{eV}$ is required. In plasmas with ITER-like wall configuration, the absence of an intrinsic radiator leads to requirement of active seeding with extrinsic impurities to achieve sufficient divertor 
radiation required for these low target temperatures.

\section{References}

[1] T. Pütterich et. al. 2010 Nucl. Fusion 50

[2] G. Federici et. al. 2003 Jour. Nucl. Mat. 313-316

[3] G.F. Matthews et. al. 2009 Phys. Scr. T138

[4] S. Brezinsek et. al. Proceedings of the $19^{\text {th }}$ PSI conference, 2010, Jour. Nucl. Mat. in press

[5] C. Giroud et. al. Proceedings of the $23^{\text {rd }}$ IAEA Fusion Energy Conference, 2010

[6] R. Simonini et. al. 1994 Contrib. Plasma Phys. 34

[7] D. Reiter, M. Baelmans, P. Börner, 2005 Fusion Sc. and Tech. 47

[8] D. Moulton et. al. 2011 Journal of Nuclear Materials

[9] P.C. Stangeby, J.D. Elder 1995 Nucl. Fusion 35

[10] W. Eckstein et. al. 1993 Nucl. Instrum. And Meth. B83

[11] A. Thoma et. al. 1997 Plasma Phys. Control. Fusion 39

[12] D. Reiser et. al. 1998 Nucl. Fusion 38

[13] T. Pütterich et. al. 2008 Plasma Phys. Control. Fusion 50

[14] J. Neuhauser et. al.1984 Nucl. Fusion 2

[15] R. Dux et. al. 2009 Jour. Nucl. Mat. 390-391 


\section{TABLE CAPTIONS}

Table1: Inner and outer strike point (ISP / OSP) electron density, $n_{e}$, and electron and ion temperatures, $T_{e}, T_{i}$, and inner and outer target (IT / OT) deuterium current, $I_{D}$, and tungsten gross erosion rate, $I_{W}$, and inner and outer mid-plane (IMP / OMP) $n_{e}, T_{e}$, and $T_{i}$, and self-sputtering fraction, $f_{\text {self-sput }}$, prompt re-deposition fracion, $f_{\text {prompt re-dep. }}$, leakage fraction, $f_{\text {leakage, }}$, and core, main SOL, and divertor SOL tungsten contamination. 


\section{FIGURE CAPTIONS}

Figure 1: W target sputtering profiles for inner target (a) and outer target (b). The low-density case is represented by circles, the medium-density case by squares, and the high-density case by diamonds. The sputtering profiles are plotted against the distance from separatrix (i.e., distance from strike point), negative values being in the private flux region. $\mathrm{Y}$-axis is the tungsten erosion flux $\left(1 / \mathrm{m}^{2} \mathrm{~s}\right)$ plotted on a $\log$-scale.

Figure 2: Tungsten concentration in core, main SOL, divertor SOL and private flux region for the three upstream density cases: $n_{e, \text { sep OMP }}=1.9^{*} 10^{19} / \mathrm{m}^{3}$ (Low), $3.4 * 10^{19} / \mathrm{m}^{3}$ (Medium), $3.6^{*} 10^{19} / \mathrm{m}^{3}$ (High)

Figure 3: Ion temperature (a), background density (b), Spitzer collision time (c), background flow velocity (d) along the magnetic field line from outer strike point to outer x-point. The values of the low-density case are represented by red, medium-density by blue, and high-density case values by black. 


\begin{tabular}{|l|l|l|l|}
\hline & Low & Medium & High \\
\hline D IT puff [electrons/s] & $5.0 \mathrm{E}+21$ & $1.1 \mathrm{E}+22$ & $1.4 \mathrm{E}+22$ \\
\hline$n_{e}\left[\mathrm{~m}^{-3}\right]$ ISP $/$ OSP & $4.7 \mathrm{E}+20 / 3.9 \mathrm{E}+19$ & $1.12 \mathrm{E}+21 / 6.7 \mathrm{E}+20$ & $1.09 \mathrm{E}+21 / 8.8 \mathrm{E}+20$ \\
\hline$T_{e}$ ISP $/$ OSP $[\mathrm{eV}]$ & $6.5 / 106.9$ & $0.8 / 7.9$ & $0.8 / 3.8$ \\
\hline$T_{i}$ ISP / OSP $[\mathrm{eV}]$ & $5.6 / 92.2$ & $0.8 / 5.5$ & $0.8 / 5.3$ \\
\hline$I_{D}$ IT / OT $\left[\mathrm{s}^{-1}\right]$ & $1.4 \mathrm{E}+23 / 7.8 \mathrm{E}+22$ & $1.5 \mathrm{E}+23 / 2.9 \mathrm{E}+23$ & $1.3 \mathrm{E}+23 / 3.1 \mathrm{E}+23$ \\
\hline$I_{W}$ IT / OT $\left[\mathrm{s}^{-1}\right]$ & $1.8 \mathrm{E}+19 / 1.2 \mathrm{E}+20$ & $0 / 3.3 \mathrm{E}+19$ & $0 / 1.2 \mathrm{E}+18$ \\
\hline$n_{e} \mathrm{IMP} /$ OMP $\left[\mathrm{m}^{-3}\right]$ & $3.4 \mathrm{E}+19 / 1.9 \mathrm{E}+19$ & $4.7 \mathrm{E}+19 / 3.4 \mathrm{E}+19$ & $4.7 \mathrm{E}+19 / 3.6 \mathrm{E}+19$ \\
\hline$T_{e}$ IMP $/$ OMP $[\mathrm{eV}]$ & $114.4 / 134.7$ & $102.5 / 115.5$ & $100.6 / 112.8$ \\
\hline$T_{i}$ IMP / OMP $[\mathrm{eV}]$ & $207.5 / 276.5$ & $148.1 / 208.6$ & $145.2 / 199.1$ \\
\hline$f_{\text {self-sput. }}[\%]$ & 19.8 & 0.72 & 0.44 \\
\hline$f_{\text {prompt } r e-d e p .}[\%]$ & 83 & 99 & 97 \\
\hline$f_{\text {leakage }}[\%]$ & 3.5 & 0.0004 & 0 \\
\hline$C_{w}$ core $\left[n_{w} / n_{e}\right]$ & $2.3 \mathrm{E}-05$ & $2.5 \mathrm{E}-11$ & $0.0 \mathrm{E}+00$ \\
\hline$C_{w}$ SOL $\left[n_{w} / n_{e}\right]$ & $1.6 \mathrm{E}-04$ & $1.2 \mathrm{E}-09$ & $0.0 \mathrm{E}+00$ \\
\hline$C_{w \text { div }}\left[n_{w} / n_{e}\right]$ & $1.8 \mathrm{E}-04$ & $5.6 \mathrm{E}-08$ & $8.5 \mathrm{E}-10$ \\
\hline & & & \\
\hline
\end{tabular}



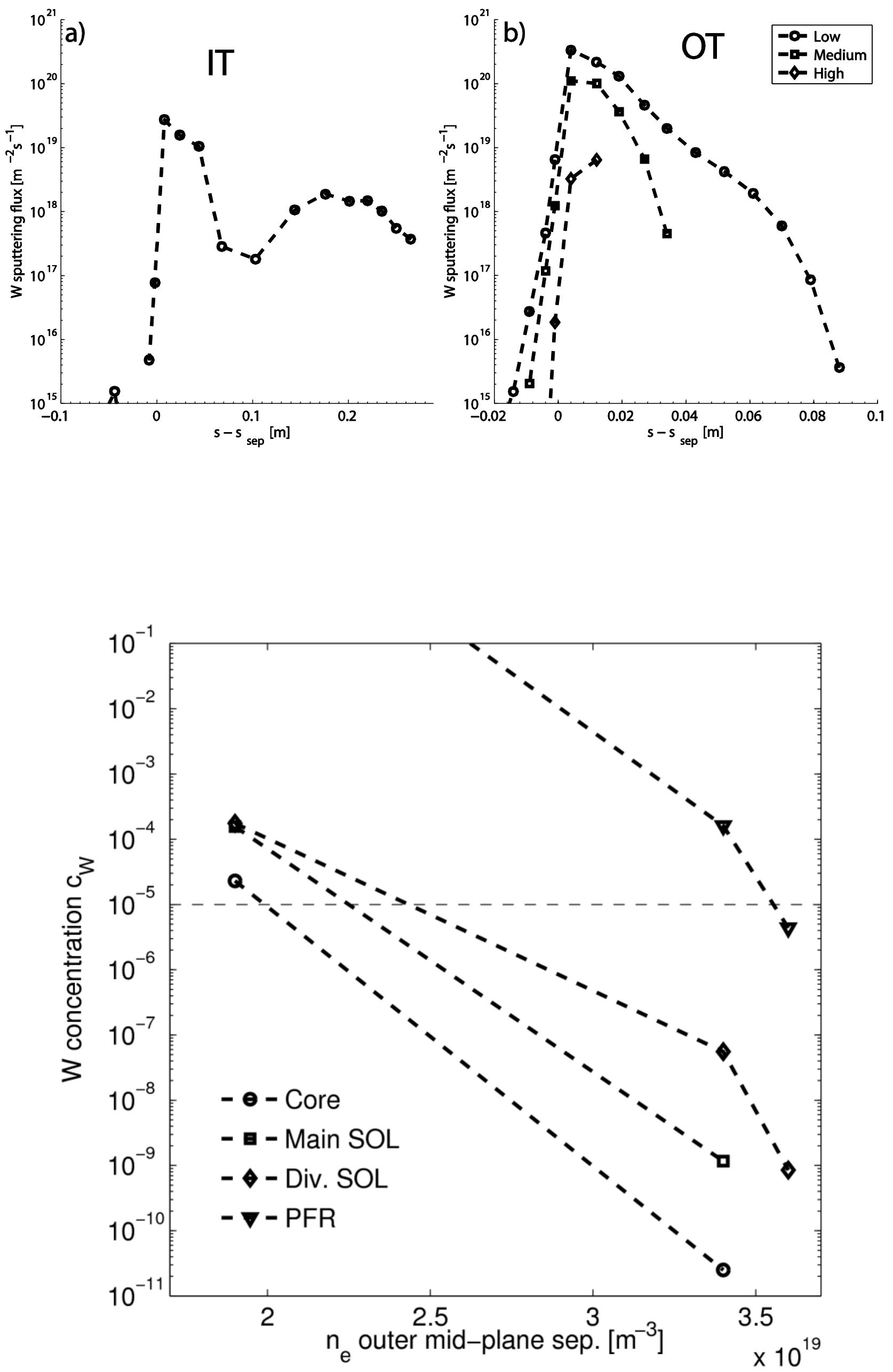


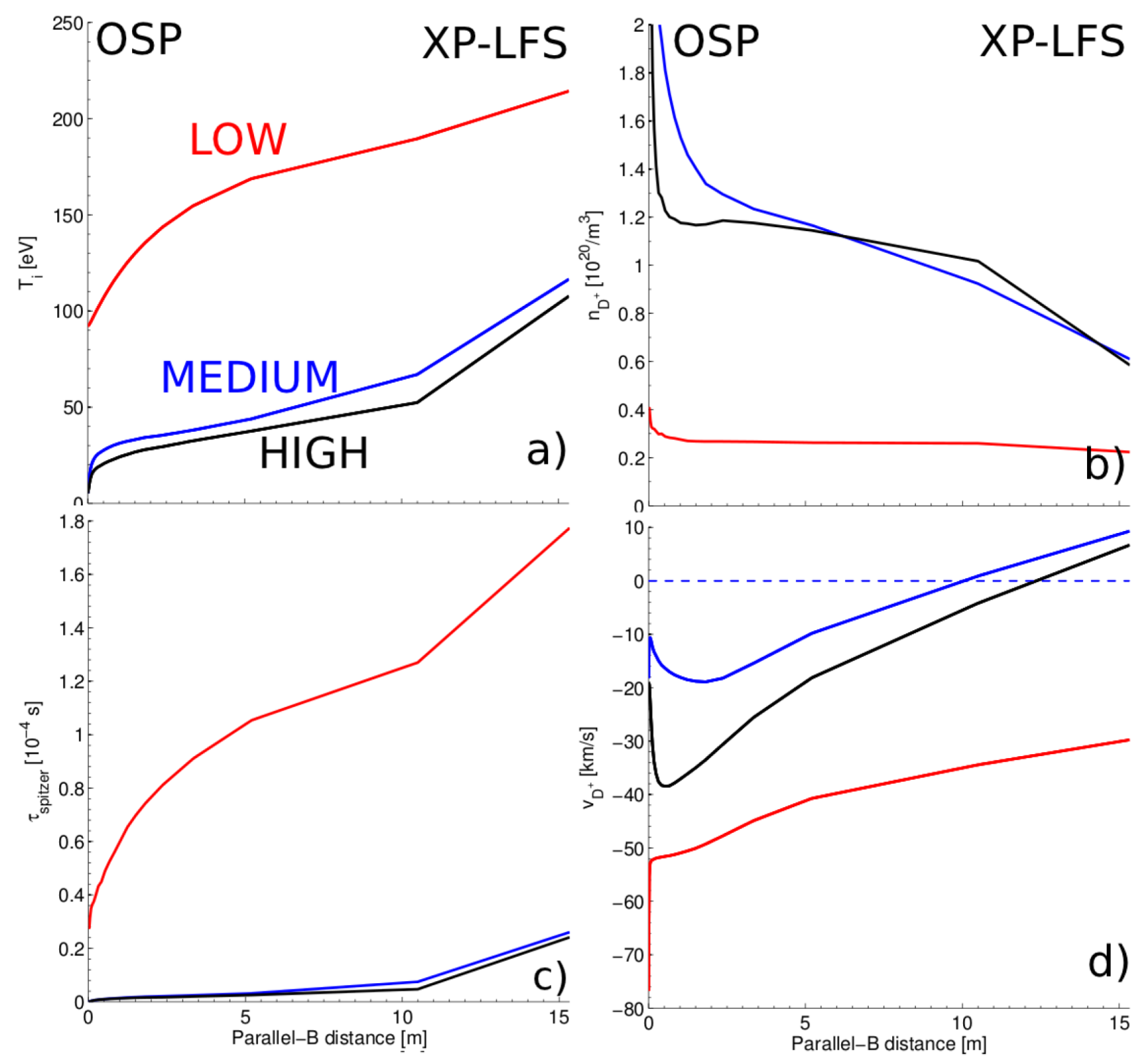

